\title{
Dermatomyositis Associated with Docetaxel Use in the Treatment for Ovarian Cancer
}

\author{
Yuta Ito, Yuko Hamada, Keiko Katayama, Hirokazu Uno, Tokio Nakada \\ Department of Dermatology, Showa University Fujigaoka Hospital, Yokohama, Japan \\ Email: tokio@med.showa-u.ac.jp
}

How to cite this paper: Ito, Y., Hamada, Y., Katayama, K., Uno, H. and Nakada, T. (2017) Dermatomyositis Associated with Docetaxel Use in the Treatment for Ovarian Cancer. Case Reports in Clinical Medicine, 6, 281-284.

https://doi.org/10.4236/crcm.2017.610031

Received: September 1, 2017

Accepted: October 20, 2017

Published: October 23, 2017

Copyright $\odot 2017$ by authors and Scientific Research Publishing Inc. This work is licensed under the Creative Commons Attribution International License (CC BY 4.0).

http://creativecommons.org/licenses/by/4.0/ (c) (i) Open Access

\begin{abstract}
A 50-year-old woman presented with a 1-week history of rash on the face and hands and difficulty when rising from a sitting position. She underwent an exploratory laparotomy for ovarian cancer 8 months ago. Three months ago, she underwent total hysterectomy, adnexectomy, and retroperitoneal lymph node dissection. Docetaxel and carboplatin were started and administered twice. Physical examination revealed periorbital edema with a purplish appearance; Erythematous lesions on radiodorsal aspects of the proximal interphalangeal and metacarpophalangeal joints were seen. We diagnosed as dermatomyositis on the basis of clinical findings. Interview demonstrated that such rashes were enhanced 1 week after chemotherapy, and got better through following one week. Cutaneous and muscular symptoms got worse two months after her initial visit. Myotonia was found in electromyography, and high density areas on both femoral regions and lower thighs were observed by MRI of skeletal muscle. Scleroderma-like lesions are known as an adverse effect to taxane. In addition, three cases of dermatomyositis due to taxane were reported in Japan. Since dermatomyositis was regarded as one of paraneoplastic syndrome generally, it was not considered as drug-induced. However, clinical course of our case suggests that docetaxel may play more important role in activity of dermatomyositis than ovarian cancer. Seeing patients treated with taxane, therefore, we have to be careful for symptoms suggestive of autoimmune diseases: not only scleroderma but dermatomyositis.
\end{abstract}

\section{Keywords}

Dermatomyositis, Taxane, Docetaxel, Ovarian Cancer

\section{Introduction}

In taxanes, chemotherapeutic agents, paclitaxel and docetaxel are widely used to 
treat several cancers. It is known that these can produce a variety of side effects, e.g. gastrointestinal symptoms, myelosuppression, and general fatigue, etc. In addition, scleroderma-like lesions can be occurred in patients with taxane treatment: 13 cases were collected in Japan [1] [2].

\section{Case Report}

A 50-year-old woman presented at the Dermatology Department with a 1-week history of rash on the face and hands in April 2014. The patient also awarded difficulty when rising from a sitting position. She underwent an exploratory laparotomy for ovarian cancer 8 months ago. Then, she had been treated with paclitaxel and carboplatin (PTX + CBDCA) chemotherapy four times. Three month ago, she underwent total hysterectomy, adnexectomy, and retroperitoneal lymph node dissection. Since she developed wheal during the 4th CBDCA + PTX therapy, docetaxel and carboplatin (DOC + CBDCA) were started and administered twice. Physical examination revealed periorbital edema with a purplish appearance; Erythematous lesions on radiodorsal aspects of the proximal interphalangeal and metacarpophalangeal joints were seen. We diagnosed as dermatomyositis on the basis of dermatological findings. CBC noted pancytopenia: WBC $1400 / \mu \ell, \mathrm{RBC} 312 \times 10^{4} / \mu \ell$, and PLT $16 \times 10^{4} / \mu \ell$. Chemistry panel were within normal limits including CK $(62 \mathrm{U} / \mathrm{\ell})$ and CRP $(0.15 \mathrm{mg} / \mathrm{dl})$. Antinuclear antibody was $\times 80$ (speckled pattern), and anti Jo-1 antibody was less than 1 . Interview demonstrated that such rashes were enhanced 1 week after DOC and CBDCA therapy, and got better through following one week. Cutaneous and muscular symptoms got worse after the 5th of DOC + CBDCA therapy (two months after her initial visit): erythematous lesions on upper eyelids and hands enlarged and got more reddish (Figure 1 and Figure 2). Dermoscopic findings of fingers

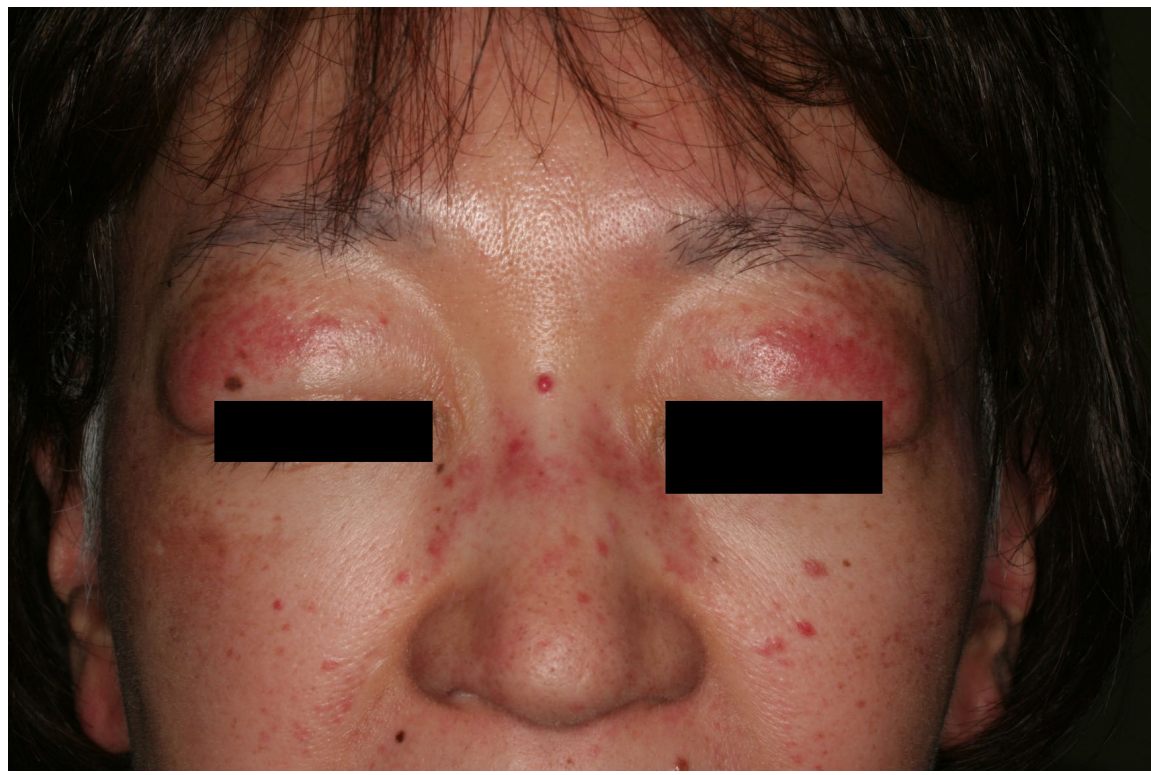

Figure 1. Clinical findings of two months after initial visit: periorbital edema with a purplish appearance. 


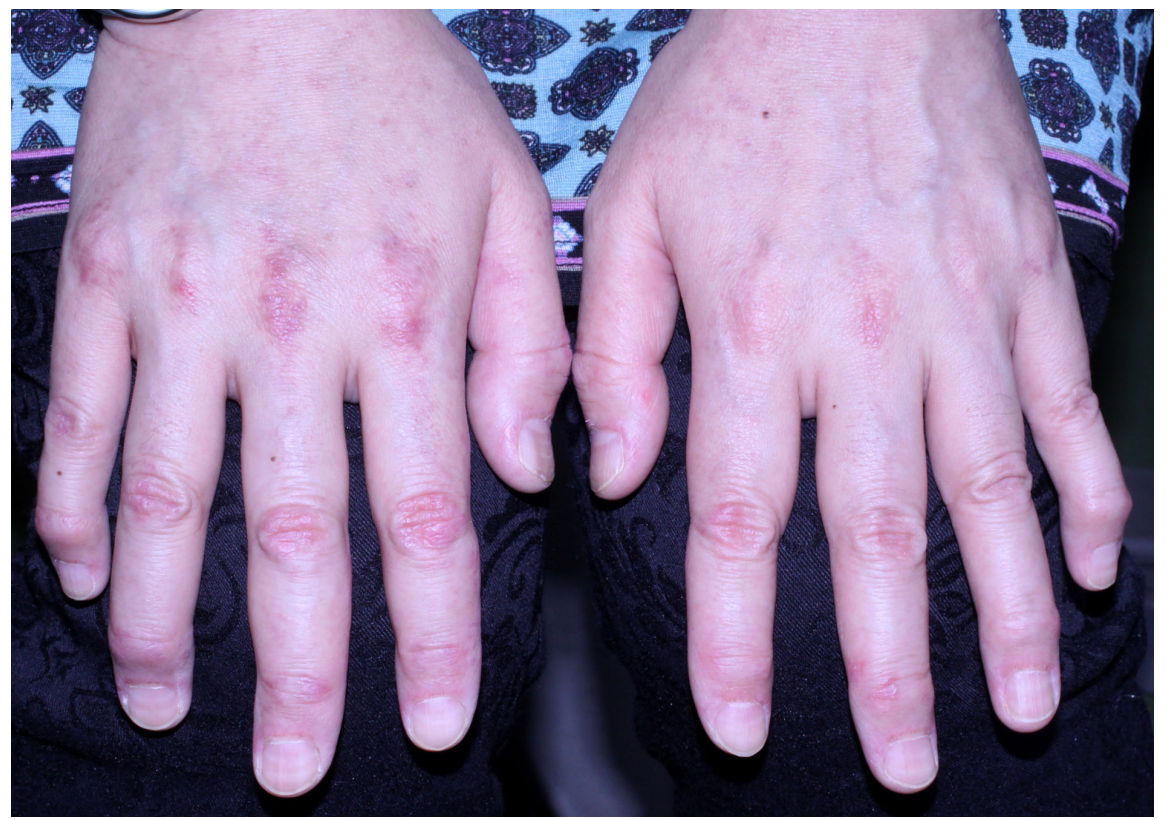

Figure 2. Erythematous lesions on radiodorsal aspects of the proximal interphalangeal and metacarpophalangeal joints.

noted nail fold capillary abnormalities: bleeding and avascular areas on eponychium. Results of blood examination showed higher CK (214 U/l) and antinuclear antibody (×640) than initial visit. Myotonia was found in electromyography, and high density areas on both femoral regions and lower thighs were observed by magnetic resonance imaging (MRI) of skeletal muscle. The patient was admitted and started on systemic corticosteroid therapy (Prednisolone, 45 $\mathrm{mg} /$ day). She was discharged on the 44th day, when $30 \mathrm{mg} /$ day of prednisolone was administered. Although dermatomyositis improved after systemic corticosteroid therapy, she died from recurrence of ovarian cancer three year and 3 months after the initial visit.

\section{Discussion}

As described above, scleroderma-like lesions can be occurred in patients with taxane treatment, and 13 cases were collected in Japan [1] [2]. In addition, three cases of dermatomyositis due to taxane were reported in our country [2] [3]. Hotta et al. reported a breast cancer patient developing dermatomyositis three weeks after docetaxel administration [3]. Since dermatomyositis was regarded as one of paraneoplastic syndrome generally, it was not considered as a drug-induced disease [4]. However, clinical course of our case suggests that DOC may play more important role in activity of dermatomyositis than ovarian cancer. It is speculated that cytokines like tumor necrosis factor (TNF)- $\alpha$ may play an important role in taxane-induced scleroderma [1]. We speculated similar mechanism: other cytokines by taxane may lead to the onset of dermatomyositis. Seeing patients treated with taxane, therefore, we have to be careful for symptoms suggestive of autoimmune diseases: not only scleroderma but dermatomyositis. 


\section{Acknowledgements}

We gratefully acknowledge the help of Professor Howard Ira Maibach, MD.

\section{References}

[1] Itoh, M., Yanaba, K., Kobayashi, T. and Nakagawa, H. (2007) Taxane-Induced Scleroderma. British Journal of Dermatology, 156, 363-367.

https://doi.org/10.1111/j.1365-2133.2006.07597.x

[2] Docetaxel (2014) Collected Report of Drug Eruptions in Japan. 16th Editon, Fukuda Dermatology Clinic, Fukuoka.

[3] Hotta, T., Hayakawa, K., Kano, Y., Shiohara, T., Nagata, Y. and Yajima, K. (2005) Dermatomyositis Arising after Docetaxel Administration in the Patient with Breast Cancer. Japanese Journal of Clinical Dermatolog, 59, 634-637.

[4] Murgic, J., Prpic, M., Kirac, I., Camino-Varela, A.M., Bolanca, A. and Kusic, Z. (2012) Dermatomyositis as Paraneoplastic Syndrome of Peritoneal and Ovarian Relapse after Long-Term Complete Remission in Patient with Metastatic Bilateral Breast Cancer. Collegium Antropologicum, 36, 325-329. 